\title{
Persistência e eficácia do regulador de crescimento pyriproxyfen em condições de laboratório para Aedes aegypti
}

\author{
Persistence and efficacy of growth regulator pyriproxyfen in laboratory \\ conditions for Aedes aegypti
}

\author{
Marcelo Carvalho de Resende ${ }^{1}$ e Renata Antonaci Gama ${ }^{2}$
}

\begin{abstract}
RESUMO
A persistência e a eficácia do regulador de crescimento pyriproxyfen foram testadas em concentrações de 0,01 e 0,05ppm, contra larvas de Aedes aegypti, utilizando os recipientes caixas d'água (45 litros), frascos de vidro (5 litros) e baldes de plástico (20 litros). As avaliações foram nos dias 1, 7, 15, 30, 45, 60, 90 e 120 após o tratamento usando larvas de $3^{\circ}$ e $4^{0}$ estádio de Aedes aegypti. Foi calculado o percentual de mortalidade de larvas, pupas e adultos, percentual de inibição de emergência de adulto e duração dos bioensaios. Observou-se que a persistência foi de 45 dias e 90 dias para concentração final de 0,01 e 0,05ppm de pyriproxyfen, respectivamente. Observamos que a mortalidade de pupas foi significativamente maior que a de larvas e de adultos para todos os recipientes e concentrações.
\end{abstract}

Palavras-chaves: Aedes aegypti. Regulador de crescimento. Pyriproxyfen.

\begin{abstract}
The persistence and efficacy of growth regulator pyriproxyfen were evaluated in two final concentrations 0.01 and $0.05 \mathrm{ppm}$ against Aedes aegypti larvae in laboratory conditions using three types of containers: cement box (45 liters), glass bottle (5 liters)and plastic bucket (20 liters). The tests were carried after 1, 7, 15, 30, 45, 60, 90 and 120 days of treatment against Aedes aegypti larvae $3^{\text {rd }}$ and $4^{\text {th }}$ instar. The percentages of larvae, pupae and adult mortality, the percentage of adult emergence inhibition and time duration of bioassays were calculated. A was observed a persistence of 45 and 90 days by using 0.01 and $0.05 \mathrm{ppm}$ final concentrations of pyriproxyfen, respectively, was observed. We observed that mortality in the pupa stage was significantly higher than larvae and adults mortality for all containers and concentrations.
\end{abstract}

Key-words: Aedes aegypti. Growth regulators. Pyriproxyfen.

0 potencial para o desenvolvimento da resistência surge como um questionamento inevitável na maioria das discussões práticas sobre a utilidade dos inseticidas nos programas de controle de vetores no Brasil. Atualmente, os inseticidas pertencentes ao grupo dos organofosforados, têm sido amplamente usados no Programa de Controle de Dengue. Como consequiência, as populações de Aedes aegypti estão sob intensa pressão de seleção de indivíduos resistentes. Devido ao aumento da resistência aos inseticidas, o uso dos mesmos deve ser meticulosamente considerado. Assim, o conhecimento e a manipulação dos fatores que podem retardar a evolução da resistência devem ser devidamente considerados nos programas de controle de vetores. Os reguladores de crescimento estão sendo testados como medidas alternativas no controle de vetores e esses reguladores atuam interferindo na pupação, desenvolvimento dos insetos, alterações na morfogênese, desenvolvimento embriológico, reprodução, diapausa, polimorfismo e comportament $0^{458}$. Os reguladores de crescimento conhecidos como terceira geração de inseticidas ${ }^{2}$, são moléculas sintéticas, análogas aos hormônios naturais dos insetos são mais seguros para a manipulação e possuem lenta evolução de resistência aos inseticidas convencionais ${ }^{1}$.

0 objetivo deste trabalho foi avaliar a persistência e eficácia, em condições de laboratório, do regulador de crescimento pyriproxyfen, em recipientes de plástico, de vidro e de cimento.

\footnotetext{
1. Laboratório de Entomologia de Minas Gerais, Belo Horizonte, MG. 2. Laboratório de Culicídeos do Departamento de Parasitologia do Instituto de Ciências Biológicas da Universidade Federal de Minas Gerais, Belo Horizonte, MG.

Endereço para correspondência: Prof. Marcelo Carvalho de Resende. Núcleo de Entomologia de Minas Gerais. R. Rio de Janeiro 1200, Centro, 30160-041 Belo Horizonte, MG.

e-mail: resendemarcelo@ig.com.br

Recebido para publicação em 14/12/2004

Aceito em 24/11/2005
} 


\section{MATERIAL E MÉTODOS}

As larvas de Aedes aegypti de $3^{\circ}$ e $4^{\circ}$ estádio foram obtidas de colônia de laboratório, de cepa suscetível, originada do laboratório da USDA de Gainesville, Flórida. Os mosquitos foram criados em condições de insetário sob controle de temperatura $\left(25^{\circ} \pm 2^{\circ} \mathrm{C}\right)$, umidade $(70-80 \%)$ e fotoperíodo (12E: $\left.12 \mathrm{~L}\right)$.

A persistência e eficácia do regulador de crescimento (IGR), pyriproxyfen (Sumilarv $0,5 \%$ G) foi avaliada em três tipos de recipientes: balde de plástico (20 litros), frascos de vidro (5 litros) e caixa de cimento ( 45 litros), prevalentes para a criação do A. aegypti em várias regiões do Brasil.

Duas concentrações finais de 0,01 e $0,05 \mathrm{ppm}$ de pyriproxyfen na formulação granulada foram ensaiadas. A pesagem e adição do pyriproxyfen nos três tipos de recipientes ocorreram no dia zero (0), onde um balde de plástico (201), uma caixa de cimento (451) e dois frascos de vidro (51) representavam um tratamento para cada dosagem e tempo de avaliação da persistência e eficácia do regulador de crescimento. Adicionalmente, para cada dosagem, tempo de avaliação e tipo de depósito, um recipiente recebeu somente água, nos quais foram tomadas amostras como controle.

Os recipientes tratados e controle ficaram expostos na parte externa do laboratório e durante o período de avaliação não houve renovação e acréscimo de água perdida por evaporação. Nos dias 1, 7, 15, 30, 45, 60, 90 e 120 dias após o tratamento, a água dos recipientes era agitada com auxilio de um bastão e retiradas amostras com um Becker. Com o auxílio de uma proveta, foram medidos $250 \mathrm{ml}$ da diluição homogeneizada do produto, dos diferentes recipientes e colocados em copos de plástico de 500ml para realização dos bioensaios.

A metodologia de bioensaios para larvas, padronizados pela Organização Mundial de Saúde (OMS), foi utilizada para realização das avaliações, sendo realizados bioensaios para cada recipiente, concentração e tempo pós-tratamento. Nos bioensaios foram utilizadas oito réplicas com 25 larvas/ réplica, totalizando 200 larvas de A. aegypti, expostas ao regulador de crescimento e quatro réplicas com 25 larvas/ réplica, totalizando 100 larvas de A.aegypti como controle.

Durante os bioensaios as larvas foram alimentadas com ração para peixes goldfish e permaneceram em condições de laboratório sendo, diariamente, registrado o número de larvas vivas e mortas. As pupas foram transferidas com auxilio de uma pipeta de Pasteur de plástico para frascos transparentes de polipropileno com capacidade para $25 \mathrm{ml}$. As pupas vivas e mortas bem como a emergência de adultos, nos tratamentos e controle, foram registradas diariamente. As leituras finalizaram no momento em que o último exemplar morreu ou sobreviveu. Com estes dados, com o objetivo de medir a eficácia e persistência de cada concentração do pyriproxyfen, os seguintes parâmetros foram determinados: a) percentual de mortalidade das larvas (mortas/ expostas); b) percentual de mortalidade de pupas (mortas/total); c) percentual de mortalidade de adultos (mortos/total); d) percentual de inibição de emergência $=[100-(100-\mathrm{E} / \mathrm{C})]$. Onde: $\mathrm{E}=\%$ emergência nos expostos; $\mathrm{C}=\%$ emergência no controle.

\section{RESULTADOS}

Inibição de emergência de adultos. Os resultados referentes à porcentagem de inibição de emergência (\%IE) para Aedes aegypti são apresentados na Tabela 1 . A inibição de emergência relaciona os adultos emergidos nos tratamentos e no controle. Para a dosagem de 0,01ppm de ingrediente ativo de pyriproxyfen, o \%IE para os três tipos de recipientes avaliados variou entre $25 \%$ e $100 \%$ durante todo o período de experimentação e que houve uma redução acentuada no \%IE para o recipiente balde de plástico (25\%) após 60 dias de tratamento e para o recipiente caixa d'água (68\%) e vidro (80\%) após 120 dias de tratamento.

Na dosagem de $0,01 \mathrm{ppm}$ de pyriproxyfen verificamos diferença significativa entre os recipientes de vidro e caixas d'água versus balde de plástico $(\mathrm{p}<0,05)$ quando analisamos o percentual de inibição de emergência de todos os tempos de pós-tratamento.

Para Aedes aegypti, na dosagem de $0,05 \mathrm{ppm}$ de pyriproxyfen, observa-se que a persistência foi significativamente diferente quando comparamos os dias pós-tratamento 1, 7, 15, 30, 45, 60,90 versus 120 dias $(\mathrm{p}<0,05)$. Na dosagem de $0,01 \mathrm{ppm}$ de pyriproxyfen, observa-se que a persistência foi significativamente diferente para os dias pós-tratamento $1,7,15,30,45$ versus 120 dias $(\mathrm{p}<0,05)$ e os dias pós-tratamento 60,90 apresentaram uma tendência de diferenciar de 120 dias.

Mortalidade de larvas, pupas e adultos. A porcentagem de mortalidade de larvas, pupas e adultos de Aedes aegypti em recipientes de plástico, vidro e cimento mantido em condições simuladas de campo e tratados com pyriproxyfen encontra-se na Tabela 2. Verifica-se para a concentração final de $0,01 \mathrm{ppm}$

\begin{tabular}{|c|c|c|c|c|c|c|c|c|c|}
\hline \multirow[t]{2}{*}{ Recipientes } & \multirow{2}{*}{$\begin{array}{l}\text { Doses } \\
(\mathrm{ppm})\end{array}$} & \multicolumn{8}{|c|}{ Dias após } \\
\hline & & 1 & 7 & 15 & 30 & 45 & 60 & 90 & 120 \\
\hline \multirow[t]{2}{*}{ Caixa d' água } & 0,01 & 100,0 & 100,0 & 100,0 & 100,0 & 100,0 & 99,0 & 92,5 & 68,0 \\
\hline & 0,05 & 100,0 & 100,0 & 100,0 & 100,0 & 100,0 & 100,0 & 100,0 & 84,5 \\
\hline \multirow[t]{2}{*}{ Frasco de vidro } & 0,01 & 100,0 & 100,0 & 100,0 & 100,0 & 100,0 & 100,0 & 98,0 & 80,0 \\
\hline & 0,05 & 100,0 & 100,0 & 100,0 & 100,0 & 100,0 & 100,0 & 98,5 & 98,0 \\
\hline \multirow[t]{2}{*}{ Balde de plástico } & 0,01 & 100,0 & 99,5 & 100,0 & 100,0 & 79,5 & 25,0 & 41,0 & 47,5 \\
\hline & 0,05 & 100,0 & 99,5 & 100,0 & 100,0 & 94,0 & 99,0 & 97,5 & 68,5 \\
\hline
\end{tabular}




\begin{tabular}{|c|c|c|c|c|c|c|c|c|c|c|}
\hline \multirow{2}{*}{$\begin{array}{l}\text { Doses } \\
(\mathrm{ppm})\end{array}$} & \multirow[t]{2}{*}{ Recipientes } & \multirow[t]{2}{*}{ Estágio } & \multicolumn{8}{|c|}{ Dias após tratamento } \\
\hline & & & 1 & 7 & 15 & 30 & 45 & 60 & 90 & 120 \\
\hline \multirow[t]{3}{*}{0,01} & caixa d'água & larva & 13,0 & 33,0 & 85,0 & 59,5 & 0,5 & 4,0 & 21,5 & 4,5 \\
\hline & frasco de vidro & & 10,5 & 47,5 & 2,5 & 39,5 & 20,5 & 3,0 & 14,0 & 7,0 \\
\hline & balde de plástico & & 11,0 & 50,5 & 4,0 & 21,5 & 0,0 & 7,0 & 15,5 & 4,5 \\
\hline \multirow[t]{3}{*}{0,05} & caixa d'água & & 17,5 & 36,0 & 86,0 & 5,0 & 2,5 & 11,5 & 25,5 & 10,0 \\
\hline & frasco de vidro & & 7,5 & 36,0 & 6,0 & 38,0 & 19,5 & 8,0 & 16,5 & 6,5 \\
\hline & balde de plástico & & 16,0 & 40,5 & 10,0 & 34,0 & 0,5 & 14,0 & 22,0 & 1,5 \\
\hline \multirow[t]{3}{*}{0,01} & caixa d'água & pupa & 78,5 & 65,5 & 15,0 & 40,5 & 92,5 & 92,5 & 48,5 & 63,5 \\
\hline & frasco de vidro & & 87,5 & 52,5 & 96,0 & 60,5 & 79,5 & 97,0 & 84,0 & 63,0 \\
\hline & balde de plástico & & 87,0 & 49,0 & 66,0 & 62,0 & 29,0 & 12,5 & 14,5 & 40,5 \\
\hline \multirow[t]{3}{*}{0,05} & caixa d'água & & 82,5 & 64,0 & 14,0 & 86,0 & 97,5 & 88,5 & 74,5 & 74,5 \\
\hline & frasco de vidro & & 92,5 & 64,0 & 94,0 & 62,0 & 80,5 & 92,0 & 82,0 & 91,5 \\
\hline & balde de plástico & & 84,0 & 59,0 & 89,0 & 64,0 & 86,5 & 84,5 & 72,5 & 30,5 \\
\hline \multirow[t]{3}{*}{0,01} & caixa d'água & adulto & 8,5 & 1,5 & 0,0 & 0,0 & 7,0 & 2,5 & 22,5 & 0,0 \\
\hline & frasco de vidro & & 2,0 & 0,0 & 1,5 & 0,0 & 0,0 & 0,0 & 0,0 & 10,0 \\
\hline & balde de plástico & & 2,0 & 0,0 & 30,0 & 16,5 & 50,5 & 5,5 & 11,5 & 2,5 \\
\hline \multirow[t]{3}{*}{0,05} & caixa d'água & & 0,0 & 0,0 & 0,0 & 9,0 & 0,0 & 0,0 & 0,0 & 0,0 \\
\hline & frasco de vidro & & 0,0 & 0,0 & 0,0 & 0,0 & 0,0 & 0,0 & 0,0 & 0,0 \\
\hline & balde de plástico & & 0,0 & 0,0 & 1,0 & 2,0 & 7,0 & 0,5 & 3,0 & 36,5 \\
\hline
\end{tabular}

de pyriproxyfen a mortalidade variou entre $0 \%$ e $85 \%$ para larva, $12,5 \%$ e $97 \%$ para pupa e $0 \%$ e 50,5\% para eclosão de adultos. Para a concentração final de $0,05 \mathrm{ppm}$ de pyriproxyfen, a mortalidade variou entre $0,5 \%$ e $86 \%, 14 \%$ e $97,5 \%, 0 \%$ e $36,5 \%$ para larvas, pupas e adultos, respectivamente. Verificamos que no estágio de pupa a mortalidade foi significativamente superior à encontrada nos estágios de larva e adulto $(\mathrm{p}<0,001)$. Analisando a mortalidade média nos estágios de Aedes aegypti e as dosagens testadas, verificamos que não houve diferença significativa na fase de larva $(\mathrm{F}=0,06, \mathrm{p}<0,05)$. Na eclosão dos adultos (dados transformados $(\log (\mathrm{Y}+1))$ a mortalidade na dosagem de $0,01 \mathrm{ppm}$ foi significativamente superior a dosagem de $0,05 \mathrm{ppm}$ de pyriproxyfen $(\mathrm{F}=2,57$, $\mathrm{p}<0,05)$. A mortalidade média de pupa foi significativamente diferente entre as dosagens testadas, onde observamos maior mortalidade média de pupas na concentração final de $0,05 \mathrm{ppm}$ de pyriproxyfen $(\mathrm{F}=2,05, \mathrm{p}<0,05)$.

Tabela 3 - Duração dos bioensaios em dias para Aedes aegypti utilizando o regulador de crescimento pyriproxyfen na concentração final de 0,01 e $0,05 \mathrm{ppm}$ de ingrediente ativo para tratamento dos recipientes caixas d'água, baldes de plástico e frascos de vidro.

\begin{tabular}{lcrrrrrrrr}
\hline Recipientes & $\begin{array}{c}\text { Doses } \\
(\mathrm{ppm})\end{array}$ & 1 & 7 & 15 & 30 & 45 & 60 & 90 & 120 \\
\cline { 3 - 10 } & 0,01 & 15 & 12 & 7 & 7 & 8 & 8 & 12 & 12 \\
\hline Caixa d'água & 0,05 & 12 & 10 & 6 & 11 & 8 & 7 & 12 & 15 \\
& controle & 12 & 10 & 10 & 9 & 8 & 7 & 12 & 15 \\
\hline Balde de plástico & 0,01 & 10 & 8 & 9 & 9 & 11 & 12 & 10 & 9 \\
& 0,05 & 10 & 7 & 8 & 9 & 11 & 12 & 10 & 10 \\
& controle & 10 & 10 & 8 & 9 & 11 & 12 & 10 & 10 \\
\hline Frasco de vidro & 0,01 & 13 & 12 & 12 & 11 & 11 & 11 & 12 & 12 \\
& 0,05 & 10 & 11 & 10 & 9 & 11 & 11 & 11 & 12 \\
& controle & 9 & 11 & 10 & 9 & 10 & 11 & 11 & 12 \\
\hline
\end{tabular}

Duração dos bioensaios. 0 tempo de duração dos bioensaios, considerado a partir do primeiro dia de leitura até que o último indivíduo morreu ou emergiu, está representado na Tabela 3. Observa-se que de acordo com 0 tipo e tempo de exposição dos recipientes, a duração média de cada bioensaio variou de no mínimo 6 e no máximo 15 dias. Não encontramos diferença significativa entre tipos de recipientes $(\mathrm{F}=2,52, \mathrm{p}>0,05)$ e tempo de exposição $(\mathrm{F}=1,82$, $\mathrm{p}>0,05)$ para $A$. aegypti na dosagem de $0,01 \mathrm{ppm}$ e $0,05 \mathrm{ppm}$ de pyriproxyfen.

\section{DISCUSSÃo}

Atualmente, o uso de produtos com baixa toxicidade, alto efeito residual e elevada eficiência, vem sendo priorizados em estudos de controle de formas imaturas de culicídeos. Os reguladores de crescimento são um desses produtos que estão sendo avaliados e utilizados em algumas regiões do Brasil e em outros países.

Nayar e $\operatorname{cols}^{7}$, ao comparar regulador de crescimento pyriproxyfen com methoprene $(1,5 \%)$ em condições de laboratório e, parcialmente, de campo nas concentrações de 0,02 e $0,05 \mathrm{ppm}$ contra larvas de $3^{\circ}$ e $4^{\circ}$ estádio de $A$. aegypti, A. albopictus, Culex nigripalpus e Aedes taeniorhynchus, observaram que em ambas concentrações, condições de experimentação e em todas as espécies, a inibição de emergência ocasionada pelo pyriproxyfen foi maior do que a do methoprene. $\mathrm{Na}$ concentração de 0,05ppm a inibição de emergência de $A$. aegypti foi maior do que a outra concentração. Esse resultado corrobora com 0 presente trabalho, no qual houve inibição de emergência maior para Aedes aegypti na concentração de $0,05 \mathrm{ppm}$ do que de $0,01 \mathrm{ppm}$ em todos os recipientes e em todos os tempos de envelhecimento. 
Mulligan e Schaefer ${ }^{6}$ utilizaram o pyriproxyfen na concentração de $0,1 \mathrm{~kg} / \mathrm{ha}$ em duas lagoas poluídas, para 0 controle de larvas de Culex sp. Eles observaram o controle da população do inseto por períodos de 7 a 68 dias, sendo o grau de poluição da mesma um fator que interferiu na redução do efeito residual do produto. Sugeriram a utilização de outros métodos de controle vetorial alternados ao uso do regulador para prevenir ou diminuir o efeito da resistência desses insetos e para eliminar a população de larvas quando o pyriproxyfen ainda não está presente em uma concentração suficiente para produzir completa mortalidade dos indivíduos.

Okazawa e cols ${ }^{8}$, com a aplicação de pyriproxyfen em criadouros temporários para Anopheles punctulatus, nas concentrações de 0,$1 ; 0,01 ; 0,02$ e $0,05 \mathrm{ppm}$, observaram completa inibição de emergência, por dois meses, na concentração de $0,1 \mathrm{ppm}$, mesmo tendo ocorrido várias inundações na área. Nessa mesma concentração, durante 0 período seco, foi observada inibição de até 50 dias. Foi verificada, ainda, maior mortalidade no estágio de pupa quando utilizadas altas dosagens e quando a aplicação do produto era recente; ao contrário, com o decorrer do tempo de ensaio a mortalidade de adultos se tornava maior. 0 mesmo resultado foi observado no presente trabalho (Tabela 2), e também por Estrada e Mulla ${ }^{3}$, que utilizaram pyriproxyfen nas concentrações de 0,0005 ; 0,001; 0,0025 e 0,005mg/litro contra larvas de Anopheles quadrimaculatus, A. aegypti e Culex tarsalis. Para larvas de $4^{\circ}$ estágio de A. aegypti, a inibição de emergência variou de 57\% (com $0,0005 \mathrm{mg} / \mathrm{l})$ a $100 \%(\operatorname{com} 0,005 \mathrm{mg} / \mathrm{l})$ e foi observada maior mortalidade acumulada no estágio de pupa que variou de $53 \%(\operatorname{com} 0,0005 \mathrm{mg} / \mathrm{l})$ a $100 \%(\mathrm{com} 0,005 \mathrm{mg} / \mathrm{l})$.

Neste trabalho, observou-se que a duração dos bioensaios não apresentou diferença significativa entre as concentrações e tempo de envelhecimento. (MLQ Pinzón, MEC Sales, EJP Perez, GLR Uribe, IDV Bernal: dados não publicados), em 2003, em Medellín, obtiveram resultados semelhantes, testando duas concentrações de pyriproxyfen, $0,01 \mathrm{e}$ $0,05 \mathrm{ppm}$, contra larvas de A. aegypti, C. quinquefasciatus e A. albimanus, sob condições de laboratório. A duração dos bioensaios variou de 6 a 38 dias, sendo as maiores durações na concentração de $0,01 \mathrm{ppm}$. A espécie $A$. aegypti foi a que demorou mais dias para morrer. Também, foi observada maior mortalidade no estágio de pupa com diferença significativa entre as concentrações de 0,01 e $0,05 \mathrm{ppm}$ para Aedes e Culex. Nesse mesmo trabalho, não foi observada diferença significativa entre as doses e a inibição de emergência de adultos para A. albimanus e C. quinquefasciatus, mas sim para $A$. aegypti, na qual a dose de $0,05 \mathrm{ppm}$ foi mais efetiva.

0 regulador de crescimento pyriproxyfen mostrou-se eficiente na inibição da emergência de adultos de A. aegypti nas duas doses testadas, com menor efeito residual na dosagem de $0,01 \mathrm{ppm}$, em 45 dias, quando comparada à dosagem de $0,05 \mathrm{ppm}$ que, por 90 dias, persistiu adequada. Assim, os autores recomendam a dosagem de aplicação na concentração final de $0,05 \mathrm{ppm}$, por um período de 90 dias.

\section{AGRADECIMENTOS}

A Sumitomo Chemical Company pelo fornecimento do produto testado, Suzana Maria Barros Rebouças e Giovani Pontel Gonçalves funcionários do Núcleo de Entomologia de Minas Gerais.

\section{REFERÊNCIAS BIBLIOGRÁFICAS}

1. Adames EA. Ecologia y Evaluacion de Sumilarv, 0,5 G (Juvenoide: Pyriproxyfen) sobre moscas (Diptera: Muscidae) en Panama, Tese de mestrado, Faculdad de Ciências Naturales y Exactas, Universidad de Panamá, Panamá, 1993.

2. Consoli RGBA, Oliveira RL. Principais mosquitos de Importância Sanitária no Brasil. Fundação Oswaldo Cruz, Rio de Janeiro, 1994.

3. Estrada JG, Mulla MS. Evaluation of two new insect growth regulators against mosquitoes in the laboratory. Journal of the American Mosquito Control Association 2:57-60, 1986.

4. Feinsod FM, Spielman A. nutrient-mediated juvenile hormone secretion in mosquitoes. Journal of Insect Physiology 26:113-117, 1980.

5. Klowden MJ, Blackmer JL. Humoral control of Pre-oviposition behaviour in the mosquito Aedes aegypti. Journal of Insect Physiology 33:689-692, 1987.

6. Mulligan FS, Schaefer CH. Efficacy of a hormone mimic, pyriproxyfen (S31183), for mosquito control in dairy wastewater lagoons. Journal of the American Mosquito Control Association 6:89-92, 1990.

7. Nayar JK, Ali A, Zaim M. Effectiveness and residual activity comparison of granular formulations of insect growth regulators Pyriproxyfen and smethoprene against Florida mosquitoes in laboratory and outdoor conditions. Journal American Mosquito Control Association 18:196-201, 2002.

8. Okazawa T, Bakotee B, Suzuki H, Kawada H, Kere N. Field evaluation of an insect growth regulator, pyriproxyfen, against Anopheles punctulatus on north Guadalcanal, Solomon Islands. Journal of the American Mosquito Control Association 7:604-607, 1991.

9. Staal GB. Insect growth regulators with juvenile hormone activity. Annual Review of Entomology 20:417-460, 1975. 